\title{
Recanalization of Intracranial Carotid Artery Occlusion Associated with Jugular Vein Cannulation
}

\author{
Ramin Zand, John K. Lynch
}

doi:10.1017/cjn.2014.21

Can J Neurol Sci. 2014; 41: 659-660

\section{INTRODUCTION}

Spontaneous recanalization of intracranial carotid artery (ICA) after an acute occlusion usually occurs early, between $6 \mathrm{~h}$ and 2 weeks, after the event. ${ }^{1,2}$ Although not well understood, the mechanism of recanalization may be due to regression of swelling after an acute intraplaque hemorrhage, existence of a residual lumen missed by imaging studies, or recanalization after thrombolysis. ${ }^{3}$ Despite substantial knowledge about the recanalization rate of intracranial vessel occlusion after intravenous tissue plasminogen activator (IV-tPA), limited information exists on the frequency of ICA recanalization after thrombolysis. In two recent studies, the rate of complete or partial ICA recanalization among patients who received IV-tPA and had ICA occlusion was 20\%-30\%. ${ }^{4,5}$ We report a case of recanalization of an extracranial ICA occlusion and subsequent reembolization of the M1 branch of middle cerebral artery (MCA) temporally related to internal jugular vein cannulation.

\section{Case Report}

A 61-year-old, right-handed, Asian man presented to the emergency department with the chief complaint of dysarthria and left-arm weakness. His symptoms started $3 \mathrm{~h}$ before his presentation. His medical history was significant for high blood pressure, which was well controlled with nifedipine. He was not on any other medications. He had a remote 10-pack-year history of tobacco use.

The review of systems was negative for chest pain, shortness of breath, neck pain, visual or hearing disturbances, dizziness, and incoordination. He otherwise reported difficulty with speech and left-arm weakness.

The examination revealed a male of average weight and height in no acute distress. His vital signs were remarkable for tachycardia (heart rate, 140-150 bpm) and low blood pressure (systolic reading, 95-105 $\mathrm{mmHg}$ ). Carotid pulses were present with no bruits. Heart auscultations revealed tachycardia with an irregular rhythm without murmur. On neurological examination, his language was appropriate but speech was dysarthric. His pupils were equal, round, and reactive to light. Extraocular movements were intact with no diplopia or nystagmus. No ptosis was evident. Mild weakness was apparent in the left-lower face. The tongue protrusion was midline and palate elevation was symmetric. The patient also had left hemiparesis with more weakness in the upper extremity than in the lower extremity. Sensory examination revealed extinction to double simultaneous stimulation. His National Institutes of Health Stroke Scale (NIHSS) was 7.
An MRI study of the brain showed a small diffusion abnormality in the right basal ganglia, with a large area of perfusion delay in the right middle cerebral artery (MCA) territory. A magnetic resonance angiography (MRA) of the head revealed decreased flow within the intracranial right ICA with cross filling of right MCA through right posterior and anterior communicating arteries (Figure 1). MRA of the neck was not performed at this time, but the study of MRI and MRA of the head source images indicated that the flow abnormality was most likely due to a proximal ICA occlusion. The patient was eligible for IV-tPA and was treated within $4 \mathrm{~h}$ of symptoms onset. He substantially improved after IV-tPA and within 20 min his symptoms resolved to an NIHSS of 1 for mild facial paresis. A follow-up brain MRI and MRA study $2 \mathrm{~h}$ after IV-tPA showed slight improvement of the perfusion abnormality in the right MCA territory but was otherwise unchanged. The patient remained clinically stable over the following $24 \mathrm{~h}$.

On the second day of hospitalization, an ultrasound-guided catheter was inserted into the right internal jugular vein. Immediately after the procedure, the patient was confused and severely dysarthric. His neurological examination showed right gaze deviation with a left superior quadrantanopia, left hemiparesis, neglect, and an NIHSS of 13. An emergent MRI and MRA revealed enlargement of the right MCA stroke with new areas of restricted diffusion within the right temporal and frontal lobes. MRA revealed flow within the right intracranial ICA not previously seen and a new occlusion of the M1 branch of right MCA (Figure 2).

His stroke evaluation was significant for high blood pressure, hyperlipidemia, and newly diagnosed atrial fibrillation. The patient was discharged on warfarin. His NIHSS at discharge was 11.

\section{DisCUSSION}

To our knowledge, no published reports exist on the recanalization of ICA occlusion after (a) passive neck movement or (b) applied compression, extracranial carotids ultrasonography, or internal jugular cannulation. Although our patient had received

From the Section on Stroke Diagnostics and Therapeutics, National Institute of Neurological Disorders and Stroke, Bethesda, Maryland, USA.

Received February 12, 2014. Final Revisions Submitted April 10, 2014 Correspondence to: Ramin Zand, National Institute of Neurological Disorders and Stroke, National Institutes of Health, Bethesda, MD 20892-1063, USA. E-mail: ramin.zand@gmail.com 


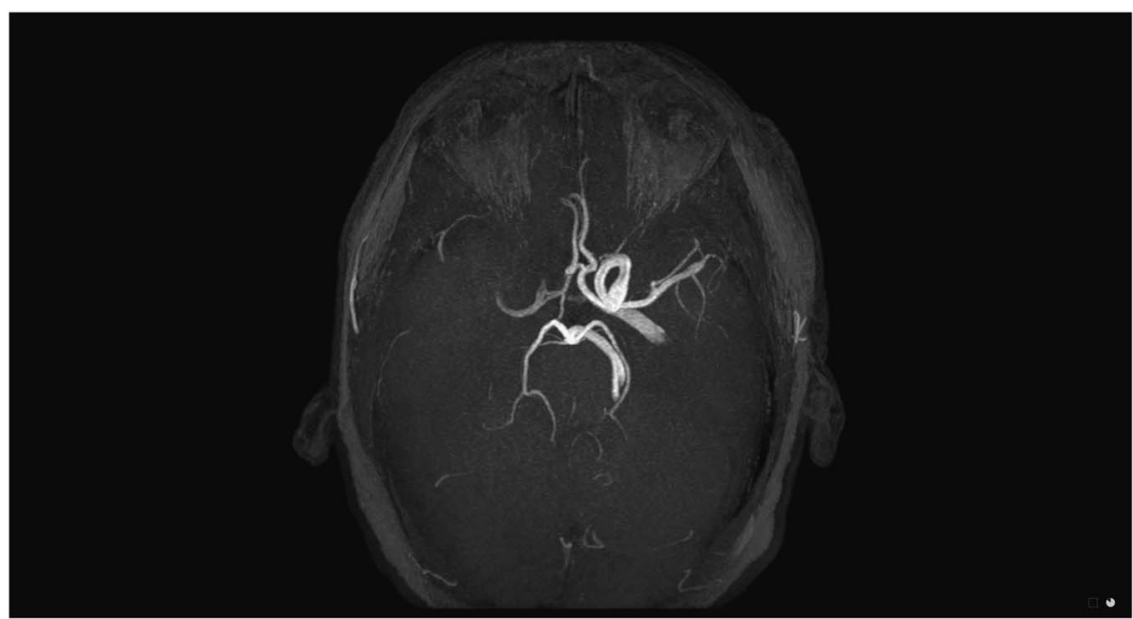

Figure 1: MRA of head before IV-tPA.

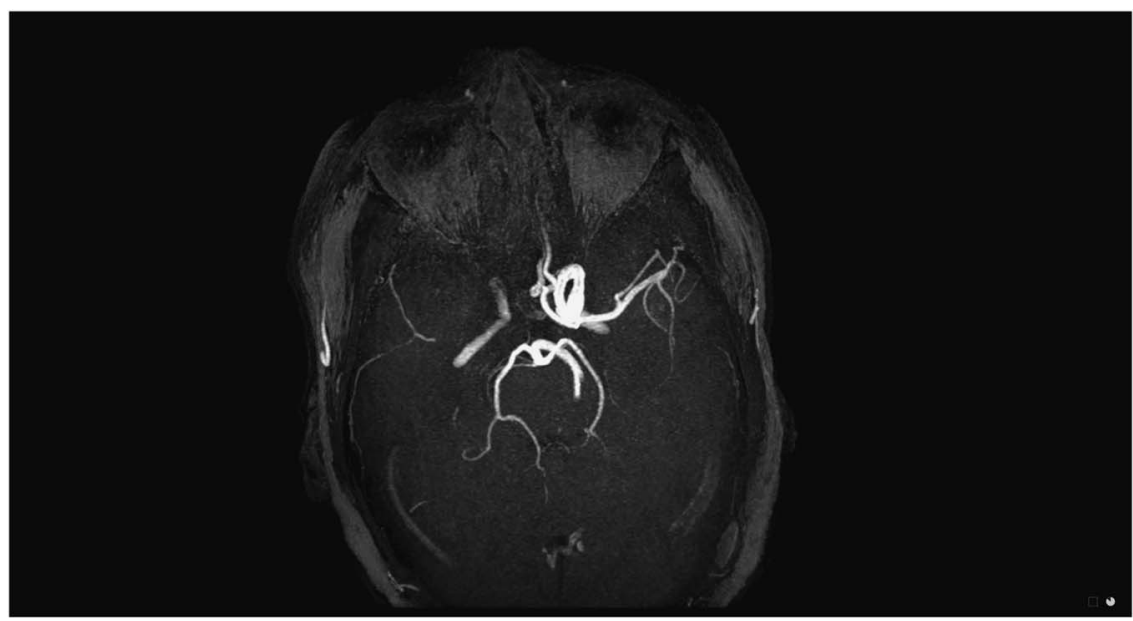

Figure 2: MRA of head shortly after right internal jugular vein cannulation.

IV-tPA $20 \mathrm{~h}$ before the incident, the temporal relationship of line placement and the development of new neurological deficits are probably related.

The central line was placed under ultrasound guidance that requires intermittent pressure to evaluate the compressibility of the vessel and to identify the internal jugular vein. This may have led to manipulation of a thrombus within the extracranial portion of ICA and embolization to the right MCA. We hypothesized that administering IV-tPA destabilized the clot. Consequently, the force due to physical maneuvers and compression of the vessels at the time of central line placement dislodged the clot and caused the reembolization of the M1 branch of MCA. Stroke neurologists and intensivists should be aware of this easily avoidable risk.

\section{REFERENCES}

1. Nguyen-Huynh MN, Lev MH, Rordorf G. Spontaneous recanalization of internal carotid artery occlusion. Stroke. 2003;34(4):1032-4.

2. Camporese G, Verlato F, Salmistraro G, Ragazzi R, Andreozzi GM. Spontaneous recanalization of internal carotid artery occlusion evaluated with color flow imaging and contrast arteriography. Int Angiol. 2003;22(1):64-71.

3. Lammie GA, Sandercock PA, Dennis MS. Recently occluded intracranial and extracranial carotid arteries. Relevance of the unstable atherosclerotic plaque. Stroke. 1999;30:1319-25.

4. Pechlaner R, Knoflach M, Matosevic B, et al. Recanalization of extracranial internal carotid artery occlusion after i.v. thrombolysis for acute ischemic stroke. PLoS One. 2013;8(1):e55318.

5. Saqqur M, Uchino K, Demchuk AM, et al. Site of arterial occlusion identified by transcranial Doppler predicts the response to intravenous thrombolysis for stroke. Stroke. 2007;38(3):948-54. 